\title{
Noninvasive cumulative axial load may induce intervertebral disc degeneration-A potential rabbit model
}

\author{
XUEDONG BAI ${ }^{1,2}$, DELI WANG ${ }^{2}$, MINGYUE ZHOU ${ }^{2}$, \\ CHENG XU ${ }^{2}$, WEI LI ${ }^{2}$, HUI TAO ${ }^{2}$, QING HE ${ }^{2}$ and DIKE RUAN ${ }^{1,2}$ \\ ${ }^{1}$ Department of Orthopedic Surgery, Xijing Hospital, The Fourth Military Medical University, Xi'an, Shaanxi 710032; \\ ${ }^{2}$ Department of Orthopedic Surgery, Navy General Hospital, Beijing 100048, P.R. China
}

Received September 23, 2015; Accepted December 19, 2016

DOI: $10.3892 / \mathrm{etm} .2017 .4148$

\begin{abstract}
Intervertebral disc degeneration (IDD) is considered to be the main cause of many spinal disorders; however, its underlying pathophysiology is not clearly understood. Recent studies indicate that excessive mechanical loading may serve a major role in the initiation of IDD. The aim of the present study was to explore the effect of noninvasive cumulative axial loading on the intervertebral discs of the lumbar spine using a novel rabbit model. Rabbits in the experimental group were placed into individual tubes specifically designed to force maintenance of an upright posture and were loaded with a heavy collar to increase the intradiscal pressure of their lumbar spine. Radiograph imaging and magnetic resonance imaging (MRI) was performed every 4 weeks to provide evidence of disc degeneration. At the end of the experiment, the animals were sacrificed and disc specimens were harvested for quantitative polymerase chain reaction and histological analysis. MRI results revealed significant and progressive reductions in the signal intensities of lumbar discs in the experimental group compared with the control group throughout the 14-week study period. The expression level of type I collagen was significantly increased and the expression levels of type II collagen and aggrecan were significantly decreased in the experimental group compared with the control group $(\mathrm{P}<0.05)$. Histological examination revealed marked structural changes in the experimental group, including fibrocartilage-like tissue ingrowth and accelerated fibrotic changes of the nucleus pulposus. The results of the present study indicate that noninvasive cumulative axial load is able to induce accelerated degenerative changes in rabbit lumbar discs, which may provide useful information for the
\end{abstract}

Correspondence to: Dr Dike Ruan, Department of Orthopedic Surgery, Navy General Hospital, 6 Fucheng Road, Beijing 100048, P.R. China

E-mail: ruandikengh@163.com

Key words: intervertebral disc degeneration, animal model, noninvasive, axial load, rabbit establishment of a novel animal model of IDD for the research of IDD in humans.

\section{Introduction}

Intervertebral disc degeneration (IDD) is associated with lower back pain, which has a significant impact on modern society $(1,2)$. The causes of IDD are multifactorial, and the underlying pathophysiology and pathogenesis are not fully understood (3). Animal models of IDD are crucial for clarifying its pathological mechanisms and provide a means for evaluating new pharmacological therapies and other treatment modalities (4). Many animal models of disc degeneration have been established; these include mechanical models such as compression or instability-induced models, structural models such as injury or chemically induced models, and models of spontaneously developing IDD (5-7). Each of these models has advantages and disadvantages when used for the study of IDD pathogenesis and/or to test novel therapies. Among these IDD models, the most commonly used is the needle puncture or stab wound-induced model $(6,8)$. All such injury-induced degeneration models require a surgical step to injure the disc and create a wound sufficient to trigger disc degeneration (9); therefore, the pathophysiology of injury-induced disc degeneration is more similar to a frustrated healing response than to the process of human disc degeneration (8). Currently, no ideal animal model for the study of IDD exists, and the identification of novel improved animal models remains of great importance (10).

Excessive mechanical loading can contribute to the initiation of IDD (11). An epidemiological study reported a positive dose-response relationship between an individual's cumulative occupational lumbar load and lumbar disc herniation, as well as lumbar disc narrowing (12). Additionally, an in vivo study investigating the effects of mechanical loading on the transport of nutrients into normal human lumbar discs demonstrated that sustained mechanical loading impairs the diffusion of nutrients into the disc and triggers changes similar to those found in disc degeneration (13). These findings suggest that a model of mechanical load-induced disc degeneration may stimulate the processes involved in human disc degeneration.

Intradiscal pressure (IDP) is thought to be one of the few determinants that indirectly influenced by axial spinal 
load (14). A previous investigation found great variations in IDP in sheep during their various daily activities. The highest IDPs (3.73-4.78 $\mathrm{MPa}$ ) were recorded when the sheep stood up from a lying position, and were 5- to 6-fold greater than the IDPs recorded while the sheep were standing (0.70-0.73 MPa) (15). This indicates that an animal's position greatly impacts its IDP, which is considered to be a significant factor influencing disc degeneration $(13,16)$.

In the present study, effects of noninvasive cumulative axial loading on the rabbit lumbar discs were explored by making rabbits maintain an upright posture and placing a noninvasive external load on the spine. The method used in this study partially simulated the excessive load-induced IDD process in humans, and thus may constitute a potential model for use in further studies.

\section{Materials and methods}

Establishment of noninvasive cumulative axial load-induced IDD model. A total of 24 male New Zealand white rabbits ( 4 months old; $2.4-2.8 \mathrm{~kg}$ ) provided by the animal center of Navy General Hospital (Beijing, China) were randomly assigned to one of two study groups: Experimental or control. Each rabbit in the experimental group $(n=12)$ was placed into a plastic tube designed to maintain the rabbit in an upright posture. The tubes were transparent, $50 \mathrm{~cm}$ in height, $14 \mathrm{~cm}$ in diameter, and equipped with steel mesh bases for ease of cleaning. While in the tubes, the rabbits were docile and unable to escape. Following a 1-week acclimation period during which rabbits in the experimental group were confined in tubes for 4-6 h a day, a 600-g collar composed of Styrofoam and plummets was placed onto the neck of each experimental rabbit to begin the formal experiment. Rabbits in the experimental group were put in their tubes for $6 \mathrm{~h}$ every day $(3 \mathrm{~h}$ in the morning and $3 \mathrm{~h}$ in the afternoon). When not confined to their tubes, the rabbits in the experimental group were housed and fed in standard cages. Rabbits in the control group $(n=12)$ were housed and fed in standard cages throughout the entire experiment. All rabbits were housed at $20-25^{\circ} \mathrm{C}, 50-60 \%$ humidity, and a $12 \mathrm{~h}$ light/dark cycle. Each rabbit was fed with $75 \mathrm{~g}$ rabbit feed, twice a day. Water was provided ad libitum. Rabbits in the two groups were weighed every 4 weeks to assess their general condition. Experimental methods were conducted in accordance with recommendations in the Guide for the Care and Use of Laboratory Animals from the National Institutes of Health (Bethesda, MD, USA). The study protocol was reviewed and approved by the Institutional Animal Care and Use Committee of Navy General Hospital (Beijing, China; no. 2013-0624).

Radiographs. Prior to the experiment, lateral radiographs were taken of each rabbit in the experimental group while the animal was sitting in an upright position in its tube to observe its body position within the tube. Rabbits in the experimental and control groups were subsequently anesthetized with xylazine (3 mg/kg intramuscularly, Shengda Pharmaceutical Co., Ltd., Jilin, China) and ketamine (40 mg/kg intramuscularly, Jiangsu Hengrui Medicine Co., Ltd., Jiangsu, China) and lumbar lateral radiographs were taken with the rabbits in the lateral decubitus position. The differences in disc height of rabbits in the experimental group were determined by measuring the disc heights on the upright and lateral decubitus radiographs.

At 4, 8 and 12 weeks following the start of the study, lumbar lateral radiographs of rabbits in the experimental and control groups were taken when rabbits were in the lateral decubitus position to examine changes in disc height. Disc heights were measured and expressed as the disc height index (DHI) using a previously reported method (6). The DHI was calculated using measurements obtained from the anterior, middle and posterior portions of the intervertebral disc, and was divided by the average of adjacent vertebral body heights. The formula $\mathrm{DHI}=2 \mathrm{x}(\mathrm{A}+\mathrm{M}+\mathrm{P}) /(\mathrm{A} 1+\mathrm{M} 1+\mathrm{P} 1+\mathrm{A} 2+\mathrm{M} 2+\mathrm{P} 2)$ was used for all radiograph measurements, where $\mathrm{A}, \mathrm{M}, \mathrm{P}$ indicate anterior, middle and posterior disc height, respectively; A1, M1, P1 indicate anterior, middle and posterior upper adjacent vertebral body height, respectively; and A2, M2, P2 indicate anterior, middle and posterior lower adjacent vertebral body height, respectively (Fig. 1D). Changes in DHI were expressed as a DHI percentage, calculated as follows: $\mathrm{DHI}(\%)=\mathrm{DHI}$ in upright position/DHI in lateral decubitus position $\mathrm{x} 100$. To evaluate differences between the lumbar discs at different levels, data from three lumbar segments were analyzed. These segments were discs L2-3, L4-5, and L6-7, representing the upper, middle and lower lumbar discs, respectively. All measurements were recorded by two independent observers, and the mean values were calculated.

Magnetic resonance imaging (MRI) examination. Rabbits in the two groups underwent MRI prior to and 4, 8, 12 and 14 weeks following commencement of the study. From 12-14 weeks, rabbits in the experimental group did not receive any treatment, and were housed and fed in the same manner as the control group while allowing time for disc recovery.

The hydration status of the nucleus pulposus (NP) was graded using a modified Schneiderman's score as previously described (17). SilverPACS-ADViewer software version 4.8 (Zhejiang SilverPAC HEA Ltd., Zhejiang, China) was used to determine grayscale values for the NP of discs of interest and cerebrospinal fluid in the same image on a T2-weighted sagittal MRI scan. The grayscale value for the NP was normalized against that of the cerebrospinal fluid, which was arbitrarily assigned a value of one. To evaluate differences among lumbar discs at different levels, the data from the three lumbar segments were analyzed. All measurements were recorded by two independent observers and the mean values were calculated.

Reverse transcription-quantitative polymerase chain reaction (RT-qPCR) analysis of gene expression. At 14 weeks, rabbits from the two groups were sacrificed by injection of $100 \mathrm{mg} / \mathrm{kg}$ pentobarbital (Sigma-Aldrich; Merck Millipore, Darmstadt, Germany). The NPs of L5-6 were obtained and immediately frozen at $-196^{\circ} \mathrm{C}$ in liquid nitrogen for subsequent RT-qPCR analysis.

Total ribonucleic acids (RNA) of tissue was extracted using TRIzol $^{\circledR}$ (Invitrogen; Thermo Fisher Scientific, Inc., Waltham, MA, USA). 2 U DNase I enzyme was mixed with $1 \mu \mathrm{g}$ extracted RNA and incubated at $37^{\circ} \mathrm{C}$ for $20 \mathrm{~min}$ to remove genomic DNA using the DNA-free ${ }^{\mathrm{TM}}$ kit (Takara Bio, Inc., Otsu, Japan), followed by inactivation of DNase using $1 \mu \mathrm{l}$ of the provided DNase inactivation reagent. RNA concentration 
and integrity were analyzed in denaturing electrophoresis gel and spectrophotometer (A260/A280 and A230/A280 ratios). RT-qPCR was conducted using PrimeScript ${ }^{\mathrm{TM}}$ RT Master Mix (Takara Bio, Inc.). Briefly, $1 \mathrm{ml}$ RNA was mixed with $2 \mathrm{ml}$ 5X PrimeScript RT MasterMix and $10 \mathrm{ml}$ RNase free $\mathrm{dH}_{2} \mathrm{O}$ was added. Mixed solutions were incubated for $15 \mathrm{~min}$ at $37^{\circ} \mathrm{C}$ and then at $85^{\circ} \mathrm{C}$ for $5 \mathrm{sec}$, and finally stored at $-80^{\circ} \mathrm{C}$ prior to qPCR. qPCR assays were performed with a SYBR Premix Ex Taq $^{\text {TM }}$ PCR kit (Takara Bio, Inc.) and a Mini Opticon Detector system (Bio Med Sciences, Inc., Allentown, PA, USA). Briefly, 10 ul SYBR-Green (containing DNA polymerase), 10 pmol forward and reverse primer, and $100 \mathrm{ng}$ cDNA were used in a final volume of $20 \mu \mathrm{l}$. All primers were designed using Premier 5.0 software (Premier Biosoft International, Palo Alto, CA, USA) and are presented in Table I. GAPDH was used as a housekeeping gene due to its stable expression in all tissue. The qPCR thermal cycling conditions were as follows: Initial denaturation at $95^{\circ} \mathrm{C}$ for $10 \mathrm{~min}$, followed by 40 cycles of denaturation at $95^{\circ} \mathrm{C}$ for $15 \mathrm{sec}$, annealing at $60^{\circ} \mathrm{C}$ for $30 \mathrm{sec}$ and extension at $72^{\circ} \mathrm{C}$ for $30 \mathrm{sec}$. Melting curve analysis was performed following the final amplification period via a temperature gradient of $95^{\circ} \mathrm{C}$ for $15 \mathrm{sec}, 60^{\circ} \mathrm{C}$ for $15 \mathrm{sec}$ and $95^{\circ} \mathrm{C}$ for $15 \mathrm{sec}$. The $\log _{2}\left(\Delta-\Delta-\mathrm{C}_{\mathrm{q}}\right)$ was performed by comparing the mean experimental $\Delta-\mathrm{C}_{\mathrm{q}}$ to the mean control animal $\Delta-C_{q}(18)$. The mean value for each sample was used in the final analysis. All the experiments were performed in triplicate.

Histological analysis. Following sacrifice, disc segment L6-7, which exhibited the most obvious degenerative changes on MRI examination, was removed using a miniature electronic saw and prepared for histological analysis. The specimens were immediately fixed in $10 \%$ formaldehyde at $25^{\circ} \mathrm{C}$ for 1 week, decalcified using Perenyi's fluid (4\% nitric acid, $0.15 \%$ chromic acid and $30 \%$ ethanol) for 6 days and washed with running water for $12 \mathrm{~h}$ to remove excess acid. Tissue samples were subsequently cut mid-sagittally, embedded in paraffin, cut into 5- $\mu \mathrm{m}$ slices, stained with hematoxylin and eosin (H\&E, 10 min hematoxylin staining and $1 \mathrm{~min}$ eosin staining) at $25^{\circ} \mathrm{C}$ and examined via light microscopy. Additional sections were stained with $0.05 \%$ picrosirius red for $30 \mathrm{~min}$ at $25^{\circ} \mathrm{C}$ and examined via polarized light microscopy to observe any changes in collagen fibers that may have occurred during disc degeneration.

Statistical analysis. All statistical analysis was performed using SPSS version 18.0 for Windows (SPSS, Inc., Chicago, IL, USA). Differences between means of data from radiographic and MRI measurements were analyzed using repeated measurement analysis of variance and Fisher's least significant differences post hoc test. The homogeneity of variances was initially assessed using Levene's test. If the variances were homogeneous, the least-significant difference test was performed; otherwise, Tamhane's T2 was used. All data are expressed as the mean \pm standard deviation. $\mathrm{P}<0.05$ was considered to indicate a statistically significant difference.

\section{Results}

General conditions. During weeks 3 and 7 of the study, two rabbits in the experimental group became agitated and died; their data were not included in the final results. The remaining 10 rabbits in the experimental group tolerated the treatment well. Throughout the 14-week experiment, the mean body weight of rabbits in the experimental and control groups increased from initial values of 2.56 and $2.62 \mathrm{~kg}$, respectively, to final values of 3.63 and $3.81 \mathrm{~kg}$, respectively. No significant differences in weight were observed between the experimental and control groups at any time point.

Radiography. A representative lateral radiograph of an experimental group rabbit being maintained in an upright position in its tube is displayed in Fig. 1A and B. In this position, there was a marked kyphotic curvature of the lower lumbar spine, and the observed disc height is markedly less than that in the image of the same rabbit in a lateral decubitus position (Fig. 1C). A schematic representation of the technique used to measure DHI is displayed in Fig. 1D. Changes in the DHI when rabbits were in an upright posture were expressed as percentage of DHI and normalized to measurements obtained while the rabbits were in the lateral decubitus position. Significant differences between the disc heights in the upright and lateral decubitus positions were observed for all of the three measured sections (all $\mathrm{P}<0.05$ ). For L2-3, the mean disc height in the upright position was $74.4 \%$ of its height in the lateral decubitus position. This ratio dropped to $60.7 \%$ for L4-5 and 46.5\% for L6-7. A significant difference in disc height reduction was also observed among different segments $(\mathrm{P}<0.05$; Fig. 1E).

Fig. 2A and B present representative lateral radiographs of rabbits from the control and experimental groups, respectively; these were obtained prior to treatment and again at 4, 8 and 12 weeks post-treatment. A steady reduction in DHI values was observed over time for the control and experimental groups; however, no significant difference was observed between the two groups at any lumbar level or at any time point (Fig. 2C-E).

MRI. Serial MRI scans of the lumbar spinal areas of animals in the experimental and control groups were obtained prior to and at 4, 8, 12 and 14 weeks following treatment. For each group, representative, T2-weighted, mid-sagittal plane images from the same rabbit at different time points are displayed in Fig. 3. Signal intensities of lumbar discs from rabbits in the experimental group decreased progressively over the 14-week experimental period, particularly in the lower lumbar discs (Fig. 3A). By contrast, NP signal intensities in the control group decreased more slowly during the same period (Fig. 3B). Grayscale values for the NP areas decreased gradually in both groups over time, in particular during the first 8 weeks. In the L2-3 segment, no significant difference was observed between the experimental and control groups at any time point (Fig. 3C). In segment L4-5, a significant reduction was observed in the experimental group compared with the control group at week 12, and was maintained over the following 2-week recovery period ( $\mathrm{P}<0.05$; Fig. 3D). In segment L6-7, a significant reduction was observed in the experimental group compared with the control group at week 8 , which persisted until the end of the experiment $(\mathrm{P}<0.05$; Fig. 3E).

$R T-q P C R$. The relative expression levels of matrix genes in the experimental and control groups were assessed by comparing 
Table I. Primer sequences.

Sequence

GeneBank

Gene

Forward: 5'-GGAGAAAGCTGCTAA-3'

L23961

GAPDH

Reverse: 5'-ACGACCTGGTCCTCGGTGTA-3'

Collagen I $\alpha$

Forward: 5'-ATGGATGAGGAAACTGGCAACT-3'

D49399

Reverse: 5'-GCCATCGACAAGAACAGTGTAAGT-3'

Collagen II $\alpha$

Forward: 5'-CCTGTGCGACGACATAATCTGT-3'

AF027122

Reverse: 5'-GGTCCTTTAGGTCCTACGATATCCT-3'

Aggrecan
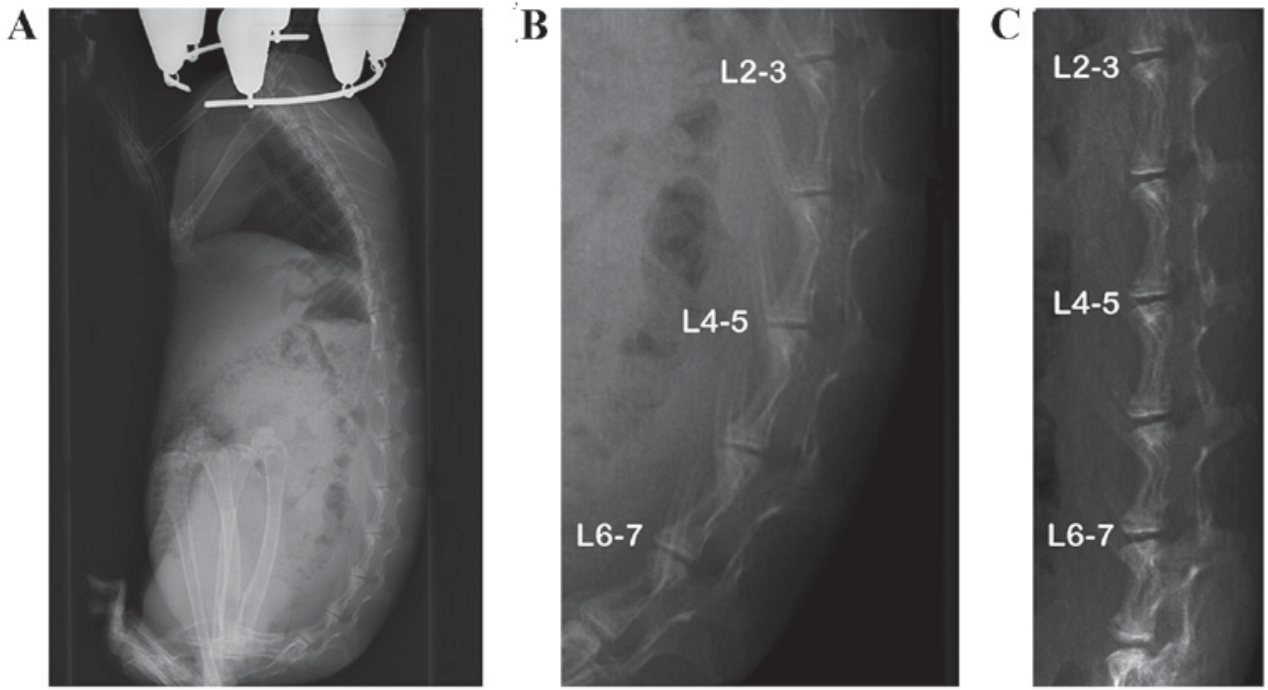

D

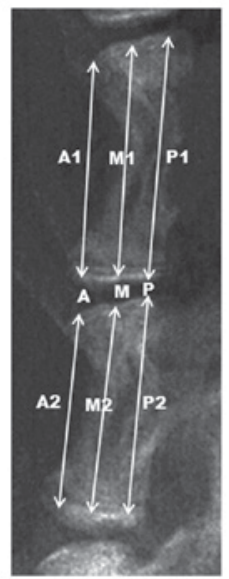

E

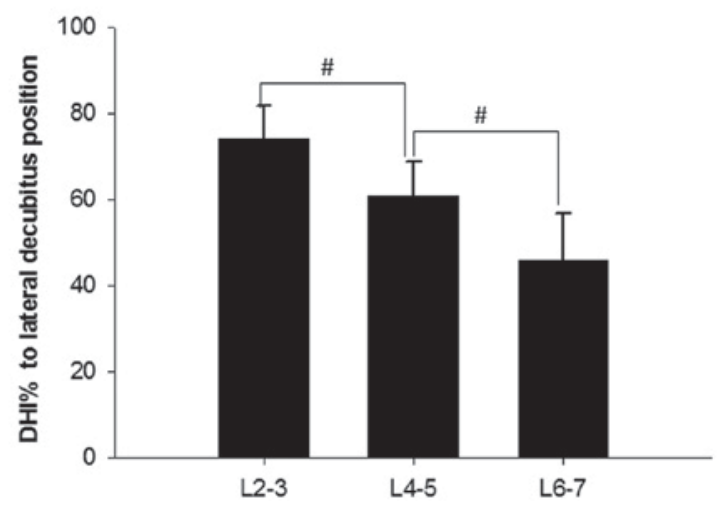

Figure 1. Body position of rabbits in the experimental group and disc height measurements under different conditions. (A) Lateral radiograph of an experimental rabbit while maintained in an upright posture by a tube, and (B) a locally enlarged lumbar image from this lateral radiograph. (C) Lateral radiograph of the same rabbit in the lateral decubitus position. (D) Schematic representation of the measurements taken to calculate the DHI. (E) Changes in the DHI, where $\mathrm{DHI} \%$ is the DHI in the upright position as a percentage of DHI in the lateral decubitus position. All data are expressed as the mean \pm standard deviation. ${ }^{\#} \mathrm{P}<0.05$. DHI, disc height index. A, M, P indicate anterior, middle and posterior disc height, respectively; A1, M1, P1 indicate anterior, middle and posterior upper adjacent vertebral body height, respectively; A2, M2, P2 indicate anterior, middle and posterior lower adjacent vertebral body height, respectively.

the mean experimental animal $\Delta-\mathrm{C}_{\mathrm{q}}$ with the mean control animal $\Delta-\mathrm{C}_{\mathrm{q}}$. The expression of collagen type I $\alpha$ mRNA was significantly higher in the experimental group compared with the control group (3.06-fold; $\mathrm{P}<0.05)$, whereas collagen type II $\alpha$ and aggrecan mRNA expression levels were significantly downregulated (0.35- and 0.37-fold, respectively; $\mathrm{P}<0.05$; Fig. 4). 

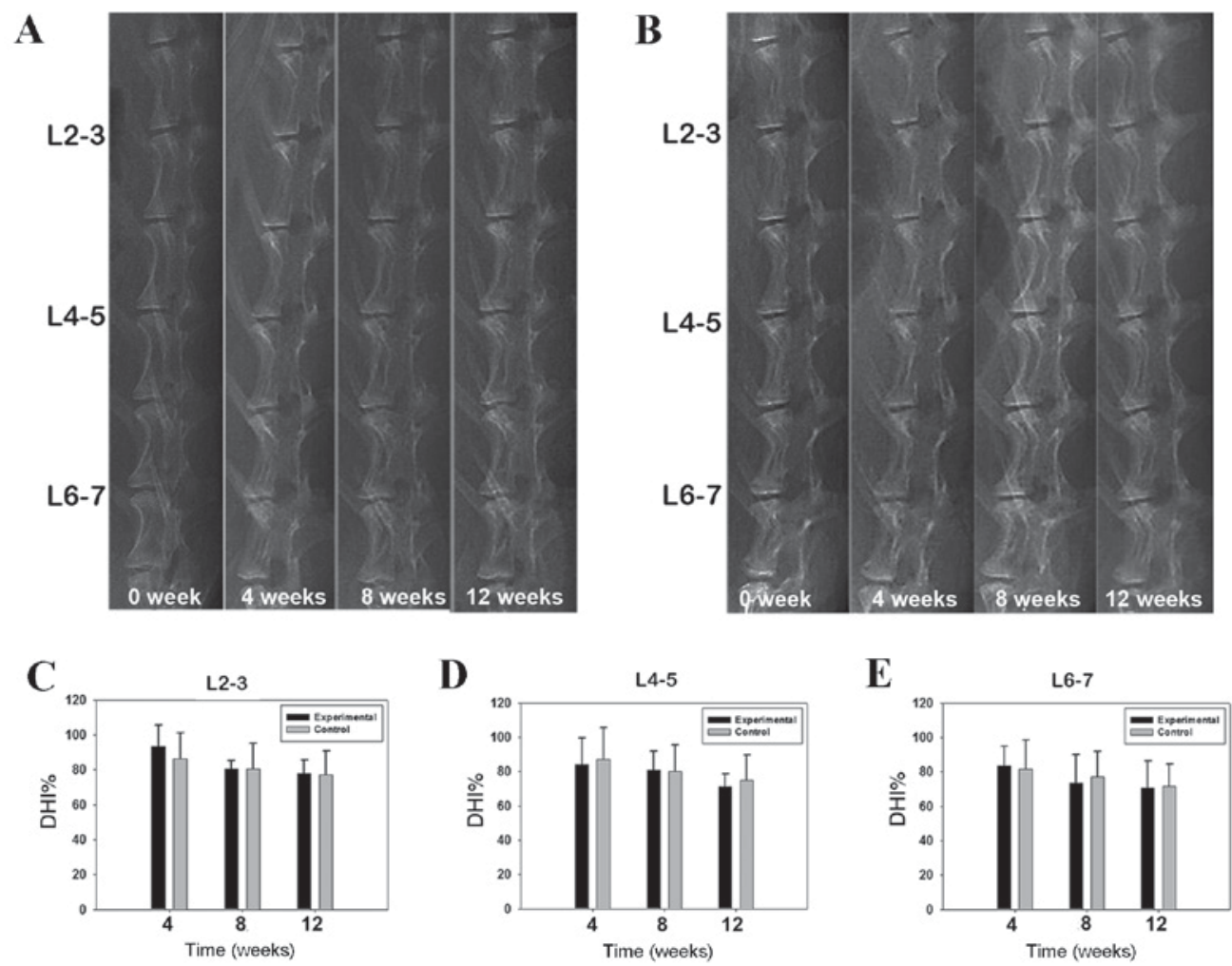

Figure 2. Serial lateral radiographs of rabbits in the control and experimental groups. (A) Control group and (B) experimental group. Changes in the DHI of (C) L2-3, (D) L4-5, and (E) L6-7 at different time points were expressed as percentage DHI and normalized to those measured prior to treatment. DHI, disc height index.

Histology. Representative histological sections of disc segment L6-7 in the midsagittal plane are displayed in Fig. 5. These include light microscopy images following H\&E staining and polarized microscopy images following picrosirius red staining. In the control group, H\&E staining revealed a clear demarcation between the NP and annulus fibrosus (AF), and scattered clusters of NP cells within an abundant gelatinous matrix (Fig. 5A). Picrosirius red staining revealed a nearly intact $\mathrm{AF}$, with a normal pattern of fibrocartilage lamellae and a well-defined border between the AF and NP (Fig. 5B). In the experimental group, H\&E staining revealed a marked increase in fibrocartilage lamellae at the inner border of the posterior $\mathrm{AF}$, resulting in a markedly decreased gelatinous NP area (Fig. 5C). Locally enlarged images (indicated by red boxes in Fig. 5C) demonstrated that the increased inner lamellae had the appearance typical of fibrocartilage, and originated from cartilage endplates (Fig. 5E), whereas the outer border of the posterior AF lost its fibrocartilage lamellar structure and resembled hyaline cartilage (Fig. 5F). Picrosirius red staining revealed newly formed birefringent collagen fibers infiltrating the NP from the margins of the cartilage endplates (Fig. 5D). The thickened posterior AF lacked the normal structure of fibrocartilage lamellae, and exhibited an uneven staining pattern (Fig. 5D).

\section{Discussion}

The present study explored the effects of noninvasive cumulative axial loading on the intervertebral discs in a rabbit model. The animals used in this study were young rabbits ( $\sim 4$ months old), which are readily obtainable by any institution. The total death rate of $16.7 \%$ appears comparable to that seen in other disc degeneration models (19). Compared with previously established disc degeneration models, the model used in the present study was implemented without producing disc injury and thus avoided surgical intervention. The loading pattern used in the present study partially mimics the cumulative effects of occupational lumbar loading in humans, which has been reported to have positive dose-response associations with lumbar disc herniation and spondylosis $(12,20)$.

To increase the IDP in lumbar discs, rabbits were placed into frictionless tubes designed to maintain them in an upright posture, and the 'body weight' above the lumbar disc was increased using a heavy collar. A pilot study revealed that rabbits were able to withstand this treatment as long as the external loading weight was $\leq 1 / 4$ of its body weight $(600 \mathrm{~g})$, and the working time was $\leq 6 \mathrm{~h}$ per day; otherwise, rabbits may experience adverse effects including irritability or anorexia. To ensure that rabbits in the experimental group were properly fed, they were allowed to eat and rest at noontime. Apart from the time spent in the tubes, the rabbits in the experimental group were housed under the same conditions as the control group.

Radiographs captured when rabbits were in the upright position revealed that the lower lumbar disc space was significantly narrowed relative to its height in the lateral decubitus position ( $>50 \%$ in disc segment L6-7), indicating increased IDP in the lumbar discs when rabbits were upright. The disc narrowing was most severe in the lower lumbar spine. This may be due in part to greater compression forces being exerted 

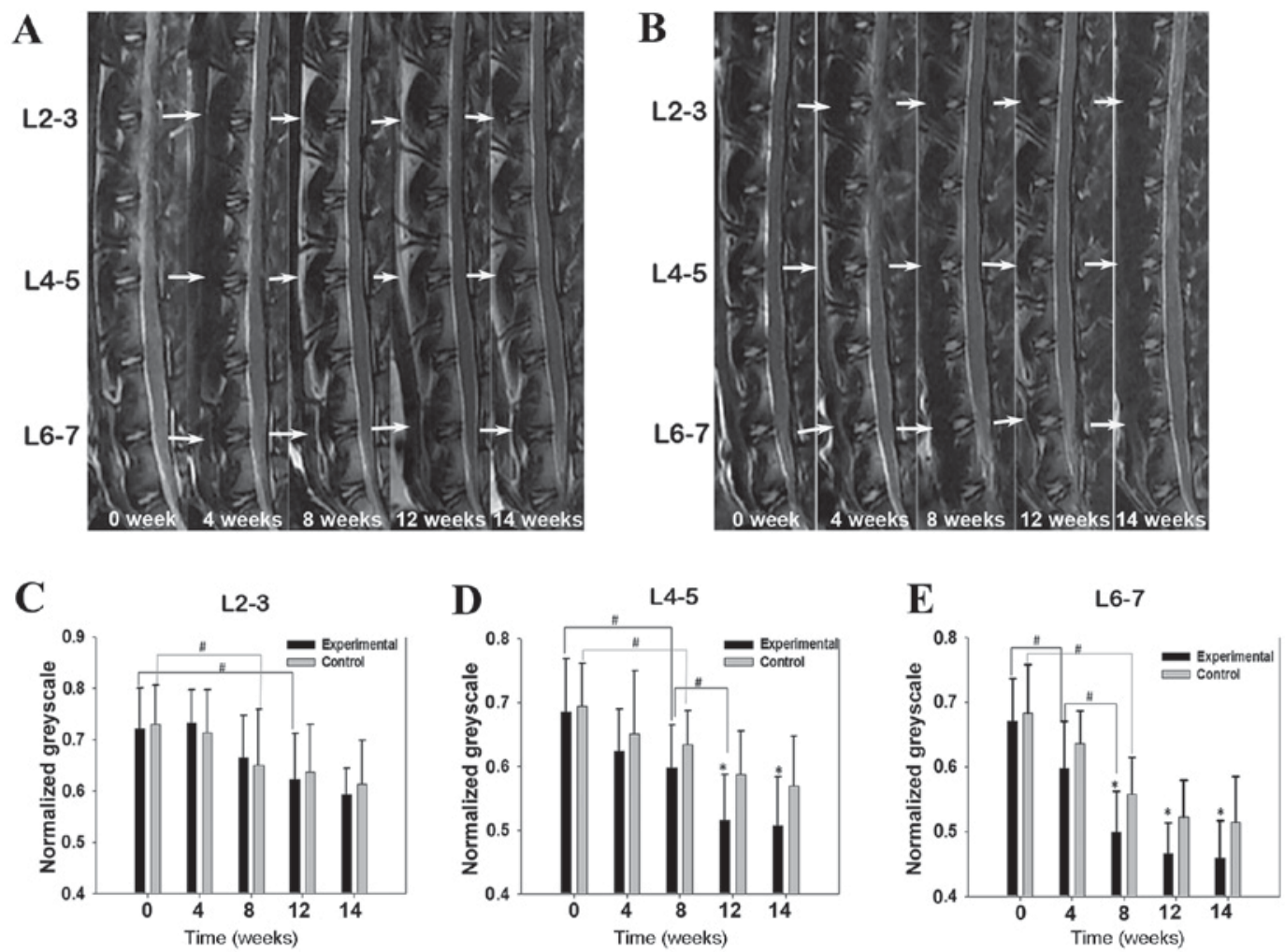

Figure 3. Serial MRI scans of the lumbar spine in the control and experimental groups. Images of a rabbit from (A) the experimental group and (B) the control group. Normalized grayscale values of the NP in the control and experimental groups were compared at disc levels (C) L2-3, (D) L4-5 and (E) L6-7. All data are expressed as the mean \pm standard deviation. ${ }^{*} \mathrm{P}<0.05$ vs. control group, ${ }^{\prime \prime} \mathrm{P}<0.05$ vs. 0 weeks. MRI, magnetic resonance imaging; NP, nucleus pulposus.

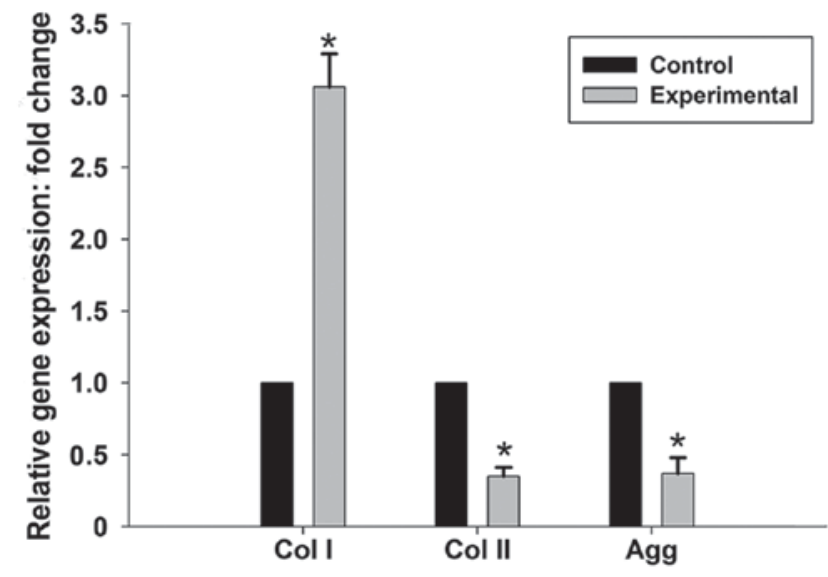

Figure 4. Quantitative relative gene expression profile of the experimental and control groups. All data are expressed as the mean \pm standard deviation. ${ }^{*} \mathrm{P}<0.05$ vs. control group. Col I, collagen I $\alpha$; Col II, collagen II $\alpha$; Agg, aggrecan.

on the lower lumbar discs; however, the primary cause may be the kyphotic curvature of the lower lumbar spine producing concentrated areas of stress and the muscle force required to stabilize the spine, which may contribute to increasing the IDP of experimental rabbits $(15,21,22)$.

With classic needle puncture-induced models of IDD a significant difference in DHI reduction between groups may be observed as early as 2 weeks post-treatment (6); however, in the present study no significant differences were observed in the DHI measurements at any disc level throughout the 12-week observation period. While a disc showing obvious signs of narrowing is considered to be severely degenerated, such a disc may not be an ideal model for investigating novel biological therapies for early stage disc degeneration (8). Therefore, the upright rabbit model using in the present study may represent an ideal research tool for assaying the effectiveness of such treatments for early and mild degeneration. It was notable that the DHI reduced obviously over time in both the experimental and the control group. This may be a result of the young age of the animals and their skeletal immaturity. The speed of intervertebral disc growth may not be as great as that of the adjacent vertebral body, resulting in a reduction in DHI during this period. However when the rabbits became skeletally mature at week 8 , the drop in DHI was not so precipitous.

On observation of the MRI images, the degenerative changes in the lower lumbar discs were more marked than those in the upper lumbar discs. This degenerative pattern is similar to that found in humans; disc degeneration in individuals typically occurs in the lower lumbar region as a result of increased mechanical loading and the structural properties of the motion segment (23). Following 2 weeks of recovery time, NPs in the experimental group did not recover their normal hydration status, which indicates that irreversible pathological changes occurred.

Matrix gene expression may vary in different IDD models. In a rabbit annular laceration model, the expression of collagen type I $\alpha$ and type II $\alpha$ were upregulated 2- to 8-fold while the decorin gene was downregulated $\sim 6$-fold compared with its expression in the control group (24). However, in another 

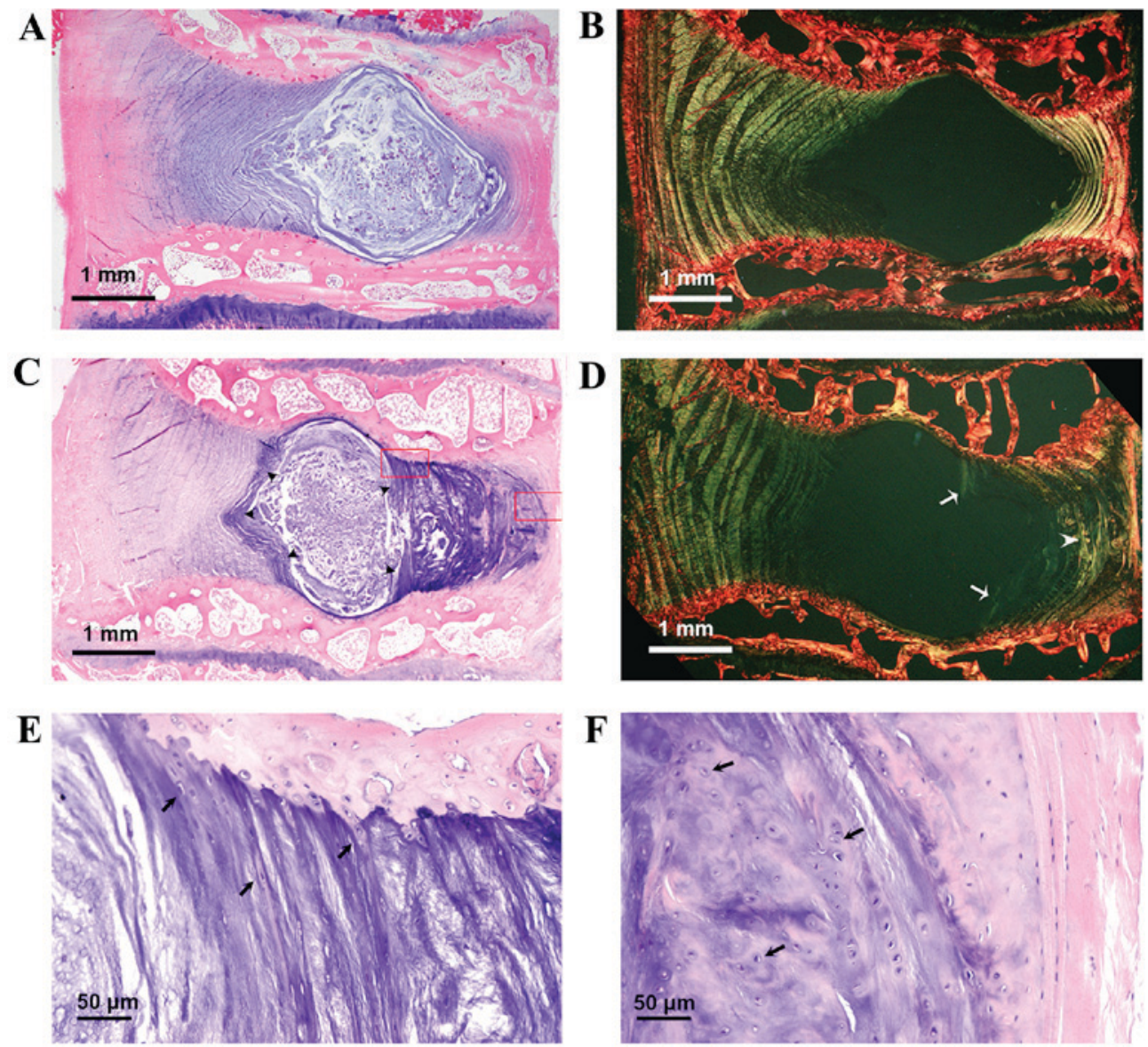

Figure 5. Histological sections of disc L6-7 in the midsagittal plane. (A) Control group with H\&E staining, exhibiting a clear demarcation between the NP and AF. (B) Control group with picrosirius red staining, revealing a normal pattern of fibrocartilage lamellae and a well-defined border between the AF and NP. (C) Experimental group with H\&E staining, revealing a large increase in fibrocartilage lamellae at the inner border of the posterior AF. Arrows indicate the distinction between the functional NP and AF. (D) Experimental group with picrosirius red staining, revealing newly formed birefringent collagen fibers infiltrating the NP from the margins of the cartilage endplates. (E) Locally enlarged image of Fig. 5C displaying the inner posterior AF in detail. Black arrows indicate increased inner lamellae, which had the appearance typical of fibrocartilage. (F) Locally enlarged image of Fig. 5C displaying the outer posterior AF in detail. Black arrows indicate the outer border of the posterior AF, which lost its fibrocartilaginous lamellar structure and resembled hyaline cartilage. H\&E, hematoxylin and eosin; NP, nucleus pulposus; $\mathrm{AF}$, annulus fibrosus.

annular stab model, aggrecan and collagen type II $\alpha$ mRNA levels decreased markedly, whereas collagen type I $\alpha$ mRNA gradually increased throughout the course of the degeneration (25). Hee et al (26) demonstrated that the collagen and glycosaminoglycan content in inner AF and NP cells cultured under 0.2 MPa compressive stress was significantly higher than that in control cells, whereas it was significantly lower than that in control cells grown under 0.4 MPa compressive stress. This indicates that the biosynthetic characteristics of human inner AF and NP cells may vary with the degree of compressive stress that they experience. The results for relative matrix gene expression in the present study are similar to those of many other load-induced degeneration models (27-29); in the experimental group the expression of collagen type I $\alpha$ mRNA was increased, whereas the mRNA expression of collagen type II $\alpha$ and aggrecan were significantly decreased compared with their respective levels in the control group. However, the examination of matrix gene expression was confined to NP tissue in the present study, and the lack of data regarding gene expression in the AF is a limitation.

It is generally believed that an increase in collagen production in the AF of discs subjected to compression may be a desirable anabolic remodeling response working to maintain the strength of the AF, whereas an increase in collagen fiber production in the nucleus may be more characteristic of an undesirable degenerative change (16). In accordance with the results of MRI and RT-qPCR, the histological examination in the present study revealed visible structural changes for the cumulative axial load-induced degeneration. Abundant fibrocartilage-like tissue grew into the NP from vertebrae endplates, dividing the NP tissue into small pieces. The NPs of the experimental group appear to have undergone accelerated fibrotic changes, which may be responsible for the dehydration changes in the MRI results. Although the widths of the posterior AFs in the experimental group appeared to be significantly increased, those AFs had lost their normal fibrocartilaginous lamellar structure, and appeared similar to hyaline cartilage (Fig. 5C and F). These structural changes may undermine the tensile strength of the posterior AFs, and may therefore be the pathological basis of disc herniation.

Polarized light microscopy was used to identify newly formed fibrocartilage containing birefringent collagen fibers (30). Picrosirius red staining followed by polarizing microscopy is able to selectively reveal collagen and allows for 
the differentiation of procollagens and pathological collagen fibers, which are not as tightly packed as normal fibers (31). In the present study, picrosirius red staining demonstrated that the distinction between the NP and AF was less marked in the experimental group compared with the control group, and that newly formed fibrous bands were included in the border of the inner AF in the experimental group. There was evidence of NPs in the experimental group having undergone accelerated 'fibrotic' changes. Such changes are considered a typical sign of disc degeneration, and are difficult to reverse (23).

The present study had some limitations that should be mentioned. The animals used were skeletally immature 4-month old rabbits and mechanical loading of their lumbar discs may not be directly comparable to the mechanical loading of skeletally mature animals or adult humans. However, degenerative changes may occur in humans prior to skeletal maturity (32); therefore, assessment of young rabbits may be relevant to early-onset degeneration. Another major limitation is that the exact in vivo IDP of rabbits in the experimental group was not measured while they were in the tubes. Additionally, it may have been possible to reduce the death rate by optimizing the loading pattern in the experiment.

In conclusion, the present study may provide a novel and feasible method for establishing a noninvasive cumulative load-induced lumbar disc degeneration model that is able to simulate the load-induced disc degeneration that occurs in humans.

\section{Acknowledgements}

The present study was supported by the National Natural Science Foundation of China (grant no. 81301579). The authors would like to thank Dr Minhua Huang and Dr Yongjun Zuo for their assistance with radiographs and MRI examinations.

\section{References}

1. Kandel R, Roberts S and Urban JP: Tissue engineering and the intervertebral disc: The challenges. Eur Spine J 17 (Suppl 4): S480-S491, 2008.

2. Shi L, Teng H, Zhu M, Li C, Huang K, Chen BI, Dai Y and Wang J: Paeoniflorin inhibits nucleus pulposus cell apoptosis by regulating the expression of $\mathrm{Bcl}-2$ family proteins and caspase- 9 in a rabbit model of intervertebral disc degeneration. Exp Ther Med 10: 257-262, 2015.

3. Issy AC, Castania V, Castania M, Salmon CE, NogueiraBarbosa MH, Bel ED and Defino HL: Experimental model of intervertebral disc degeneration by needle puncture in Wistar rats. Braz J Med Biol Res 46: 235-244, 2013.

4. Chan DD, Khan SN, Ye X, Curtiss SB, Gupta MC, Klineberg EO and Neu CP: Mechanical deformation and glycosaminoglycan content changes in a rabbit annular puncture disc degeneration model. Spine (Phila Pa 1976) 36: 1438-1445, 2011.

5. Lotz JC: Animal models of intervertebral disc degeneration: Lessons learned. Spine (Phila Pa 1976) 29: 2742-2750, 2004.

6. Masuda K, Aota Y, Muehleman C, Imai Y, Okuma M, Thonar EJ, Andersson GB and An HS: A novel rabbit model of mild, reproducible disc degeneration by an annulus needle puncture: Correlation between the degree of disc injury and radiological and histological appearances of disc degeneration. Spine (Phila Pa 1976) 30: 5-14, 2005.

7. Sun F, Qu JN and Zhang YG: Animal models of disc degeneration and major genetic strategies. Pain Physician 16: E267-E275, 2013.

8. Xi Y, Kong J, Liu Y, Wang Z, Ren S, Diao Z and Hu Y: Minimally invasive induction of an early lumbar disc degeneration model in rhesus monkeys. Spine (Phila Pa 1976) 38: E579-E586, 2013
9. Elliott DM, Yerramalli CS, Beckstein JC, Boxberger JI, Johannessen W and Vresilovic EJ: The effect of relative needle diameter in puncture and sham injection animal models of degeneration. Spine (Phila Pa 1976) 33: 588-596, 2008.

10. Sakai D: Future perspectives of cell-based therapy for intervertebral disc disease. Eur Spine J 17 (Suppl 4): S452-S458, 2008.

11. Stefanakis M, Luo J, Pollintine P, Dolan P and Adams MA: ISSLS Prize winner: Mechanical influences in progressive intervertebral disc degeneration. Spine (Phila Pa 1976) 39: 1365-1372, 2014.

12. Seidler A, Bergmann A, Jäger M, Ellegast R, Ditchen D, Elsner G, Grifka J, Haerting J, Hofmann F, Linhardt O, et al: Cumulative occupational lumbar load and lumbar disc disease-results of a German multi-center case-control study (EPILIFT). BMC Musculoskelet Disord 10: 48, 2009.

13. Arun R, Freeman BJ, Scammell BE, McNally DS, Cox E and Gowland P: ISSLS Prize Winner: What influence does sustained mechanical load have on diffusion in the human intervertebral disc?: An in vivo study using serial postcontrast magnetic resonance imaging. Spine (Phila Pa 1976) 34: 2324-2337, 2009.

14. Guehring T, Unglaub F, Lorenz H, Omlor G, Wilke HJ and Kroeber MW: Intradiscal pressure measurements in normal discs, compressed discs and compressed discs treated with axial posterior disc distraction: An experimental study on the rabbit lumbar spine model. Eur Spine J 15: 597-604, 2006.

15. Reitmaier S, Schmidt H, Ihler R, Kocak T, Graf N, Ignatius A and Wilke HJ: Preliminary investigations on intradiscal pressures during daily activities: An in vivo study using the merino sheep. PLoS One 8: e69610, 2013.

16. Iatridis JC, MacLean JJ, Roughley PJ and Alini M: Effects of mechanical loading on intervertebral disc metabolism in vivo. J Bone Joint Surg Am 88 (Suppl 2): S41-S46, 2006.

17. Ruan D, He Q, Ding Y, Hou L, Li J and Luk KD: Intervertebral disc transplantation in the treatment of degenerative spine disease: A preliminary study. Lancet 369: 993-999, 2007.

18. Livak KJ and Schmittgen TD: Analysis of relative gene expression data using real-time quantitative PCR and the 2(-Delta Delta C(T)) Method. Methods 25: 402-408, 2001.

19. Sobajima S, Kompel JF, Kim JS, Wallach CJ, Robertson DD, Vogt MT, Kang JD and Gilbertson LG: A slowly progressive and reproducible animal model of intervertebral disc degeneration characterized by MRI, X-ray, and histology. Spine (Phila Pa 1976) 30: 15-24, 2005.

20. Seidler A, Bolm-Audorff U, Heiskel H, Henkel N, Roth-Küver B, Kaiser U, Bickeböller R, Willingstorfer WJ, Beck W and Elsner G: The role of cumulative physical work load in lumbar spine disease: Risk factors for lumbar osteochondrosis and spondylosis associated with chronic complaints. Occup Environ Med 58: 735-746, 2001.

21. Beckstein JC, Sen S, Schaer TP, Vresilovic EJ and Elliott DM: Comparison of animal discs used in disc research to human lumbar disc: Axial compression mechanics and glycosaminoglycan content. Spine (Phila Pa 1976) 33: E166-E173, 2008.

22. Inoue $\mathrm{N}$ and Espinoza Orías $\mathrm{AA}$ : Biomechanics of intervertebral disk degeneration. Orthop Clin North Am 42: 487-499, 2011.

23. Adams MA, Mcnally DS and Dolan P: 'Stress' distributions inside intervertebral discs. The effects of age and degeneration. J Bone Joint Surg Br 78: 965-972, 1996.

24. Anderson DG, Izzo MW, Hall DJ, Vaccaro AR, Hilibrand A, Arnold W, Tuan RS and Albert TJ: Comparative gene expression profiling of normal and degenerative discs: Analysis of a rabbit annular laceration model. Spine (Phila Pa 1976) 27: 1291-1296, 2002.

25. Sobajima S, Shimer AL, Chadderdon RC, Kompel JF, Kim JS, Gilbertson LG and Kang JD: Quantitative analysis of gene expression in a rabbit model of intervertebral disc degeneration by real-time polymerase chain reaction. Spine J 5: 14-23, 2005.

26. Hee HT, Zhang J and Wong HK: An in vitro study of dynamic cyclic compressive stress on human inner annulus fibrosus and nucleus pulposus cells. Spine J 10: 795-801, 2010.

27. Paul CP, Schoorl T, Zuiderbaan HA, Zandieh Doulabi B, van der Veen AJ, van de Ven PM, Smit TH, van Royen BJ, Helder MN and Mullender MG: Dynamic and static overloading induce early degenerative processes in caprine lumbar intervertebral discs. PLoS One 8: e62411, 2013.

28. Liang QQ, Cui XJ, Xi ZJ, Bian Q, Hou W, Zhao YJ, Shi Q and Wang YJ: Prolonged upright posture induces degenerative changes in intervertebral discs of rat cervical spine. Spine (Phila Pa 1976) 36: E14-E19, 2011 
29. Yurube T, Takada T, Suzuki T, Kakutani K, Maeno K, Doita M Kurosaka $\mathrm{M}$ and Nishida K: Rat tail static compression mode mimics extracellular matrix metabolic imbalances of matrix metalloproteinases, aggrecanases, and tissue inhibitors of metalloproteinases in intervertebral disc degeneration. Arthritis Res Ther 14: R51, 2012.

30. Kim KW,Lim TH, Kim JG, Jeong ST, Masuda K and An HS: The origin of chondrocytes in the nucleus pulposus and histologic findings associated with the transition of a notochordal nucleus pulposus to a fibrocartilaginous nucleus pulposus in intact rabbit intervertebral discs. Spine (Phila Pa 1976) 28: 982-990, 2003.
31. Hirshberg A, Sherman S, Buchner A and Dayan D: Collagen fibres in the wall of odontogenickeratocysts: A study with picrosirius red and polarizing microscopy. J Oral Pathol Med 28: 410-412, 1999.

32. Sowa G, Vadalà G, Studer R, Kompel J, Iucu C, Georgescu H, Gilbertson L and Kang J: Characterization of intervertebral disc aging: Longitudinal analysis of a rabbit model by magnetic resonance imaging, histology, and gene expression. Spine (Phila Pa 1976) 33: 1821-1828, 2008. 